OPEN ACCESS

Edited by:

Fang Du,

Beijing Forestry University, China

Reviewed by: Liangsheng Zhang, Zhejiang University, China

Sunil Kumar Sahu, Beijing Genomics Institute (BGl),

China

Guangpeng Ren, Lanzhou University, China

${ }^{*}$ Correspondence: Xiangling You

youxiangling@nefu.edu.cn

tThese authors have contributed equally to this work

Specialty section: This article was submitted to Plant Bioinformatics, a section of the journal Frontiers in Plant Science

Received: 26 November 2021 Accepted: 14 February 2022

Published: 01 March 2022

Citation:

Liu W, Guo W, Chen S, Xu H, Zhao Y, Chen S and You X (2022) A HighQuality Reference Genome Sequence and Genetic Transformation System of Aralia elata.

Front. Plant Sci. 13:822942. doi: 10.3389/fpls.2022.822942

\section{A High-Quality Reference Genome Sequence and Genetic Transformation System of Aralia elata}

\author{
Wenxuan Liu'ti, Wenhua Guo ${ }^{2 \dagger}$, Song Chen ${ }^{1}$, Honghao $\mathrm{Xu}^{2}$, Yue Zhao ${ }^{2}$, Su Chen ${ }^{1}$ and \\ Xiangling You ${ }^{2 *}$
}

'State Key Laboratory of Tree Genetics and Breeding, Northeast Forestry University, Harbin, China, ${ }^{2}$ Key Laboratory of Saline-Alkali Vegetation Ecology Restoration, Ministry of Education, Northeast Forestry University, Harbin, China

Aralia elata is a perennial woody plant of the genus Aralia in the family Araliaceae. It is rich in saponins and therefore has a wide range of pharmacological effects. Here, we report a high-quality reference genome of $A$. elata, with a genome size of $1.21 \mathrm{~Gb}$ and a contig $\mathrm{N} 50$ of $51.34 \mathrm{Mb}$, produced by PacBio HiFi sequencing technology. This is the first genome assembly for the genus Aralia. Through genome evolutionary analysis, we explored the phylogeny and whole genome duplication (WGD) events in the A. elata genome. The results indicated that a recent WGD event occurred in the $A$. elata genome. Estimation of the divergence times indicated that the WGD may be shared by Araliaceae. By analyzing the genome sequence of $A$. elata and combining the transcriptome data from three tissues, we discovered important genes related to triterpene saponins biosynthesis. Furthermore, based on the embryonic callus induction system of $A$. elata established in our laboratory, we set up the genetic transformation system of this plant. The genomic resources and genetic transformation system obtained in this study provide insights into $A$. elata and lays the foundation for further exploration of the $A$. elata regulatory mechanism.

Keywords: Aralia elata, genome assembly, evolutionary analysis, terpenoid biosynthesis pathway, transgenic system

\section{INTRODUCTION}

Aralia elata (Miq.) Seem. (Araliaceae), also known as Chinese angelica-tree, is widely distributed in Northeast China (mainly Heilongjiang and Jilin province), Korea, Japan, Russia, the south of Far East, the south of Sakhalin, and Kuril Islands (Ahn, 1998; Reunov et al., 2007). It is one of the most desirable mountain wild vegetables in Asia benefitting from the rich nutrients in its young shoots (Cheng et al., 2021). Furthermore, as a Chinese traditional medicinal plant, A. elata plays roles on rheumatism, diabetes, hepatitis, neurasthenia, and stomach spasms (Zhang et al., 2018), especially the anti-tumor role (Duan et al., 2019). Those medicinal potentials were depended on the bioactive components of saponins. More than 100 kinds of saponins belonging to triterpene saponins have been reported in A. elata and they are mainly oleananetype saponins (Cheng et al., 2021). 
The triterpenoid biosynthesis is initiated from isopentenyl diphosphate (IPP) that is derived from the metabolism of cytosolic mevalonic acid (MVA) or the plastid methylerythritol phosphate (MEP; Sawai and Saito, 2011). This biosynthesis process is catalyzed by a series of key enzymes. The enzymes before triterpenoid structural skeleton formation include farnesyl diphosphate (FPP) synthase (FPS), squalene synthase (SS), and squalene epoxidase (SE). Oxidosqualene cyclases (OSCs) catalyze oxidosqualene to different triterpenoid backbones. $\beta$-AeAS has been identified to encode the OSC in A. elata (Wu, 2011). Subsequently, the key enzymes are cytochrome P450 monooxygenases (P450), which mediate oxidations. Uridine diphosphate-dependent glycosyl transferases (UDT) finally catalyze glycosylations to generate different triterpenoid saponins (Sawai and Saito, 2011). Recent studies revealed that subfamilies of CYP450, such as CYP71, CYP72, CYP88, CYP93, CYP716, and CYP749 are extensively involved in the oxidative stress response (Heitz et al., 2012) and the biosynthesis of triterpenes (Carelli et al., 2011; Fukushima et al., 2011; Han et al., 2011), sterols, indole alkaloids (Irmler et al., 2000; Collu et al., 2001; Nafisi et al., 2007), geraniol iridoid (Höfer et al., 2013), etc.

In $A$. elata, genes that potentially encode these key enzymes, including AeFPS (Wu, 2011), AeSS (Cheng, 2011), AeSE (Zhao, 2012), and $\beta$-AeAS (Wu, 2011) have been cloned and investigated by real-time qRT-PCR. In addition, 254 members of P450 and 122 UGT families were identified by the RNA-sequencing analysis (Cheng et al., 2020). But for the complex pathway of triterpenoid synthesis, the information of these key enzyme encoding genes is still limited due to the lack of a genome reference of this species.

With the rapid development of sequencing technology and reduction of sequencing cost, more and more plant genomes have been sequenced and published. The thirdgeneration sequencing, especially the High Fidelity ( $\mathrm{HiFi}$ ) technology, has greatly reduced the cost and shortened the circle of genome sequencing. In this study, we sequenced, assembled, and annotated a high-quality genome of A. elata using HiFi data. This is the first genome of the genus Aralia. Using comparative genomics, we explored the evolutionary trajectory and whole genome duplication (WGD) events of A. elata. We also identified the key enzyme encoding genes involved in the triterpenoid biosynthesis pathway in the genome. The expressional patterns of these genes were preliminarily investigated. Genetic transformation is the most efficient way to further explore the functions of the annotated genes in A. elata. However, no transformation system has been established for this non-model plant species. We therefore established an Agrobacterium tumefaciens mediated genetic transformation system for $A$. elata, which laid a solid foundation for plant genetic engineering. The genomic resources of $A$. elata provided here will be valuable for biological and breeding research on Aralia species and will provide new tools for Araliaceae geneticists and breeders.

\section{MATERIALS AND METHODS}

\section{Plant Materials, DNA Extraction, and Library Construction}

Fresh and healthy leaves of 2-month-old tissue culture plantlets of A. elata were harvested and immediately frozen in liquid nitrogen and preserved at $-80^{\circ} \mathrm{C}$. The samples were then sent to the company (Annoroad Gene Technology, China) for DNA extraction. The quality and quantity of the isolated DNA were assessed using a NanoDrop 2000\&8000 spectrophotometer and a Qubit 2.0 Fluorometer, respectively. Illumina and PacBio libraries were constructed using the eligible DNA following the instruction for each technology, respectively.

\section{Genome Sequencing, Assembly, and Quality Assessment}

We integrated Illumina HiSeq and PacBio $\mathrm{HiFi}$ sequencing data to achieve the complete genome sequence of $A$. elata. The Illumina library was sequenced on the Illumina HiSeq $\mathrm{X}$ Ten platform following standard Illumina protocols. After filtering out adapter sequences, low-quality reads, and duplicated reads, the clean reads were used to investigate the genomic features including genome size and heterozygosity by $k$-mer distribution analysis using GenomeScope (Vurture et al., 2017). Two libraries were constructed for PacBio HiFi sequencing. The subreads generated from the PacBio libraries were assembled into contigs using hifiasm with the default parameters (Cheng et al., 2008). The Illumina sequencing reads were aligned to the genome assembly using BWA (Li, 2013) to assess its completeness. Benchmarking Universal Single-Copy Orthologs (BUSCO) was also used to assess the quality of the final genome assembly (Simão et al., 2015).

\section{Genome Annotation}

The A. elata genome was annotated by the integration of multiple strategies including de novo, homology-based, and transcriptome-based predictions. Repeat Masker and Repeat Modeler were used to identify the repetitive sequences in the genome based on repeat sequence database. Augustus was used for de novo prediction of protein coding genes based on the repeat masked genome. For similarity-based gene prediction, eight species including Arabidopsis thaliana, Oryza sativa, Daucus carota, Populus trichocarpa, Apium graveolens, Vitis vinifera, Panax notoginseng, and Coriandrum sativum were selected, and the protein sequences of these species were downloaded from Phytozome. ${ }^{1}$ Annotation of coding genes in the genome was subsequently performed using these homologous proteins. BLAST with identity $\geq 0.95$ and coverage $\geq 0.90$ as thresholds was used to identify genes with significant similarity in the $A$. elata genome. To carry out the RNA-Seq aided gene prediction, we downloaded the transcriptome data of $A$. elata from NCBI SRA database (BioProject: PRJNA555256). The clean reads were assembled into transcripts using Trinity (Haas et al., 2013), which were aligned against the genome assembly for gene

${ }^{1}$ https://phytozome-next.jgi.doe.gov/ 
structure prediction using Program to Assemble Spliced Alignments (PASA; Haas et al., 2008). The gene sets predicted by the various strategies were integrated into a non-redundant and more complete gene set by Evidence Modeler (EVM; Haas et al., 2008). BUSCO was used to evaluate the integrity and completeness of the predicted gene set.

\section{Analysis of Genomic Evolution and WGD Events}

We used OrthoFinder to identify the orthologous groups in 12 species: five species from Apiales including A. elata, Panax notoginseng, Daucus carota, Apium graveolens, and Coriandrum sativum, two species from Asterales including Lactuca sativa and Taraxacum mongolicum, one species from Tubiflorae (Capsicum annuum), three other dicot species including Arabidopsis thaliana, Carica papaya and Populus trichocarpa, and one monocot Oryza sativa, which was used as the outgroup (Guo et al., 2021). MUSCLE was used for multiple sequence alignment for each single-copy orthologous group identified by OrthoFinder (Emms and Kelly, 2019). All the alignment blocks were then manually concatenated, and substitution model for each alignment block was estimated using ModelTest-NG (Darriba et al., 2020) program. The results were subsequently used to construct a phylogenetic tree using maximum-likelihood algorithm. Divergence times of these species in the phylogenetic tree were estimated with MCMCtree (v4.0) using the Bayesian Relaxed Molecular Clock (BRMC) approach (Yang, 2007). The parameters of MCMCtree were set as follows: burn-in=2,000; sample-frequency $=10$; and sample-number $=20,000$. Oryza sativa was designated as an outgroup of the phylogenetic tree. The calibration times of each divergent nodes were obtained from the TimeTree website (Kumar et al., 2017). Gene family amplification and contraction was analyzed by CAFÉ using the phylogenetic tree and gene numbers in each orthogroup (De Bie et al., 2006).

\section{Identification and Tissue Specific Expression of Genes Involved in Triterpene Saponins Biosynthesis}

BLASTP, with E-value of $1 \mathrm{e}-5$ as a threshold, was used to identify candidate enzymes that catalyze triterpene saponins biosynthesis. The NCBI Conserved Domain Database (MarchlerBauer et al., 2010) was used to scan conserved domains in these candidates. Only the protein sequences containing canonical domains were identified as authentic enzymes. IQ-TREE (Nguyen et al., 2015) was used to construct phylogenetic trees for these protein sequences. The expressional profiles of genes encoding these enzymes were investigated using RNA-seq data retrieved from public databases (PRJNA555256). The gene expression analysis was performed using the nf-core/rnaseq v3.2 (Ewels et al., 2020) pipeline in nextflow v21.04.1 (Di Tommaso et al., 2017). The sequencing reads were mapped to the reference genome using Spliced Transcripts Alignments to a Reference V2.7.6a (STAR) as an aligner. Gene expression levels were then determined by using RNA-Seq by ExpectationMaximization v1.3.1 (RSEM). Trimmed mean of M value (TMM;
Robinson and Oshlack, 2010) method was used to normalize and measure the expression levels of these samples.

\section{Establishment of Agrobacterium Mediated Genetic Transformation for Aralia elata}

The system of vegetable propagation of A. elata was built in our lab (Dai et al., 2011). The somatic embryogenic callus was induced from the roots of the somatic embryo plants in the induction medium $(1 / 2 \mathrm{SH}$ medium with $3.0 \mathrm{mg} / \mathrm{L}$ of IBA and $0.2 \mathrm{mg} / \mathrm{L}$ of $\mathrm{KT}$ ) for 3 weeks. When the callus was transformed to the re-differentiation medium: $1 / 2 \mathrm{SH}$ with $1.0 \mathrm{mg} / \mathrm{L} \mathrm{IBA}$ and $0.2 \mathrm{mg} / \mathrm{L} \mathrm{KT}$, after 6 weeks, lots of somatic embryo or plants emerged.

The roots of the above somatic embryo plants were used for Agrobacterium tumefaciens mediated genetic transformation. After 3 days of pre-culture, the roots were infected by $A$. tumefaciens for 5, 10, and $15 \mathrm{~min}$, respectively and then co-cultured in medium for 3 days. Next, they were transformed to the selection medium with $50 \mathrm{mg} / \mathrm{L}$ kanamycin and $200 \mathrm{mg} / \mathrm{L}$ timentin. Then after 8 weeks, the calli were checked by PCR using the primers 5'-CGC ACA ATC CCA CTA TCC TT-3', and $5^{\prime}$-AAG ACC GGC AAC AGG ATT C-3' to choose the callus line of gene transformation. The positive callus lines were transformed into above plant-medium.

\section{RESULTS}

\section{Genome Sequencing, Assembly, and Annotation}

To investigate the genomic features of A. elata, 17, 21, 25, and $27 \mathrm{~K}$-mer distribution analysis was performed (Figure 1A; Supplementary Figure 1), respectively, using $56.89 \mathrm{~Gb}$ of the Illumina reads. The Illumina reads representing $50.79 \times$ coverage based on the estimated genome size of $1.12 \mathrm{~Gb}$ ( $K$-mer analysis; Supplementary Table 1). The $K$-mer distributions followed a Poisson distribution, with two peaks corresponding to homozygous and heterozygous sequences, respectively (Supplementary Figure 1). According to the $K$-mer distribution analysis, the genome size of $A$. elata was estimated as $1.08-$ $1.14 \mathrm{~Gb}$ and the heterozygosity ratio of the genome was estimated as $1.60-1.69 \%$ (Supplementary Table 2). The results indicated that the genome of $A$. elata is highly heterozygous and repetitive. We then used HiFi technologies to sequence the A. elata genome. A total of $51.14 \mathrm{~Gb}$ of $\mathrm{HiFi}$ reads from two libraries were obtained for the genome assembly. A total of 25.75 and $25.39 \mathrm{~Gb}$ data were generated from the two libraries, respectively (Supplementary Table 3). The HiFi reads were assembled into contigs using hifiasm. The final assembled genome was $1.21 \mathrm{~Gb}$ in size with a contig N50 length of $51.34 \mathrm{Mb}$. The genome assembly contained 1,350 contigs, the longest contig was $100.88 \mathrm{Mb}$, and the average contig length was $0.89 \mathrm{Mb}$. The GC content of the A. elata genome is $36.13 \%$ (Table 1).

To evaluate the completeness of the genome assembly, short reads generated for the genomic survey were mapped to the genome. In total, $99.95 \%$ of the short reads were mapped to 


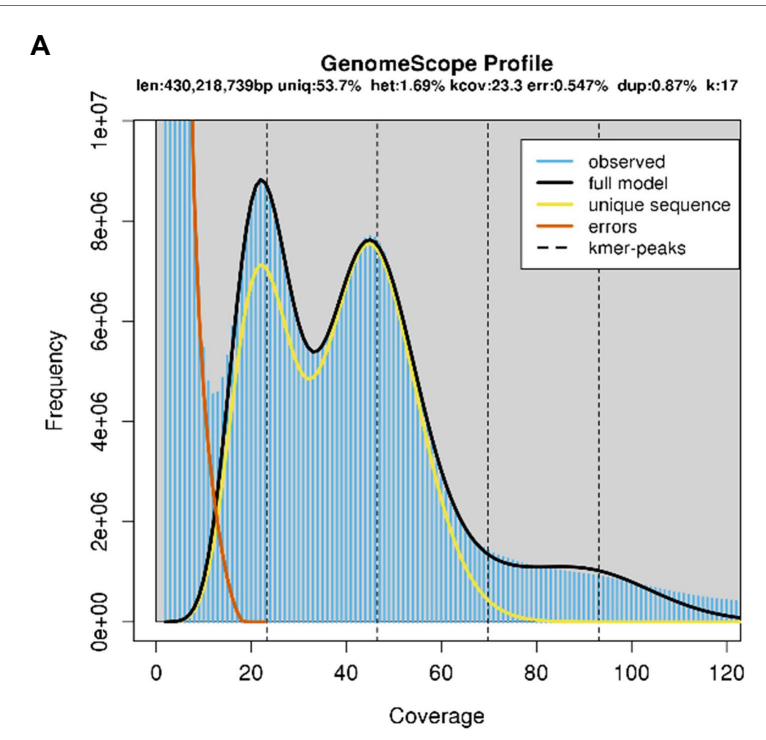

FIGURE 1 | (A) 17-mer analysis for estimating the genome size of Aralia elata. (B) Benchmarking Universal Single-Copy Orthologs (BUSCO).
TABLE 1 | Assembly statistics of the Aralia elata genome.

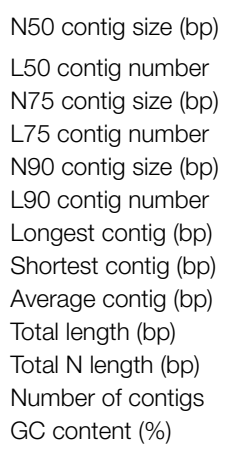
GC content (\%)

B

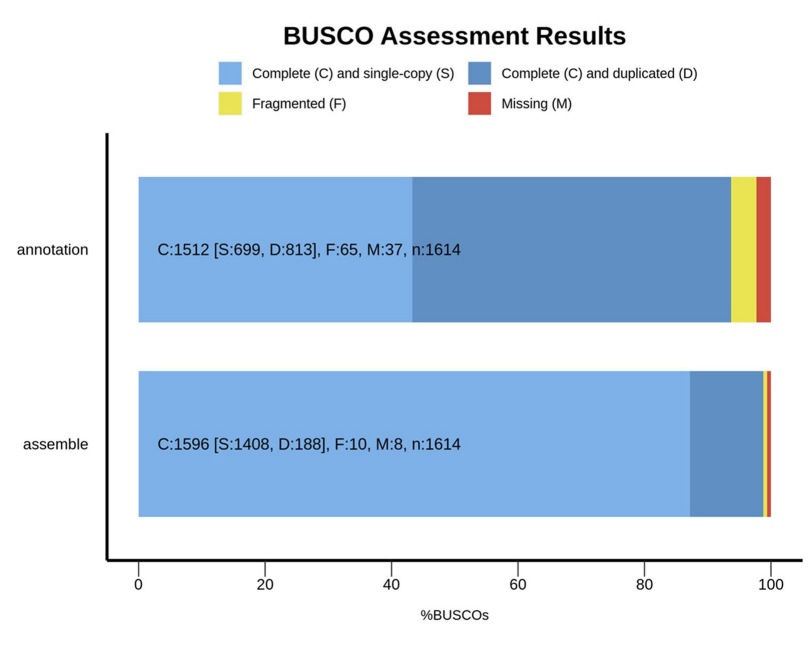

Assessment the gene coverage rate of genome assembly and annotation using

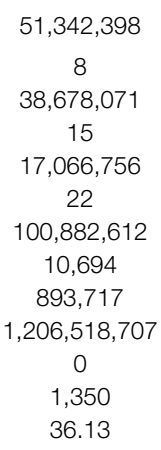

the contigs, $99.48 \%$ of which were properly pair-end mapped (Supplementary Table 4). The completeness of the genome assembly was also evaluated by BUSCO. The result revealed that the genome covered at least $98.8 \%$ of the BUSCO genes, $87.2 \%$ of which were classified as "complete and single-copy," $11.6 \%$ as "complete and duplicated," $0.6 \%$ as "fragmented", and $0.6 \%$ as "missing" (Figure 1B; Supplementary Table 5). All the results suggested a high quality of the A. elata genome assembly.

Repetitive sequences, including tandem repeat and interspersed repeats, are important parts of genomes. In this study, two strategies, de novo prediction and homology-based identification, were used to annotate the repetitive sequences in the A. elata genome. According to the integrated results obtained above, the proportion of repetitive sequences in the genome was $71.69 \%$, which was higher than carrot $(45.95 \%$; Iorizzo et al., 2016). The most abundant type of repetitive elements was long terminal repeat (LTR), which accounted for $49.15 \%$ of the genome, while DNA transposon repetitive sequences accounted for only $3.86 \%$ of the genome (Supplementary Table 6).
To annotate the protein coding genes in the A. elata genome, we used a combination of $a b$ initio prediction, homology-based search, and transcript evidence from RNA-seq data. Finally, a total of 37,016 genes were annotated in the genome. We evaluated the completeness and quality of the annotated proteome through BUSCO using Embryophyta_odb10 as database. The results indicated that $97.7 \%$ of the conserved genes were annotated in the genome, which included 93.7 and $4.0 \%$ complete and fragmented BUSCO genes, respectively. The BUSCO assessment indicated that the annotation of genome was of high accuracy (Figure 1B; Supplementary Table 7).

\section{Genome Evolution of Aralia elata}

In order to reveal the evolutionary position of $A$. elata, we compared the genome assembly with genomes from 11 other plants. A total of 250 single-copy gene families were identified among these species by OrthoFinder. These singlecopy genes were used to construct a phylogenetic tree using a maximum likelihood method. Consistent with Angiosperm Phylogeny Group, A. elata was closed to P. notoginseng, another Araliaceae species and these two species were classed into a clade. This clade was most closely related to the species from Apiales family (Figure 2A). The divergent times of these species were then estimated based on the phylogenetic tree. We estimated that A. elata and $P$. notoginseng diverged from Apiaceae at approximately 80.1 million years ago (mya). Aralia elata and $P$. notoginseng subsequently diverged into two species at around 24.2 mya. These results showed that the relationship between A. elata and $P$. notoginseng is very close. In addition, we performed a comparative analysis of gene family evolution in the phylogenetic tree. A total of 1,925 gene families were expanded in the $A$. elata lineage, whereas 1,832 gene families had undergone contraction (Figure 2A). 
A

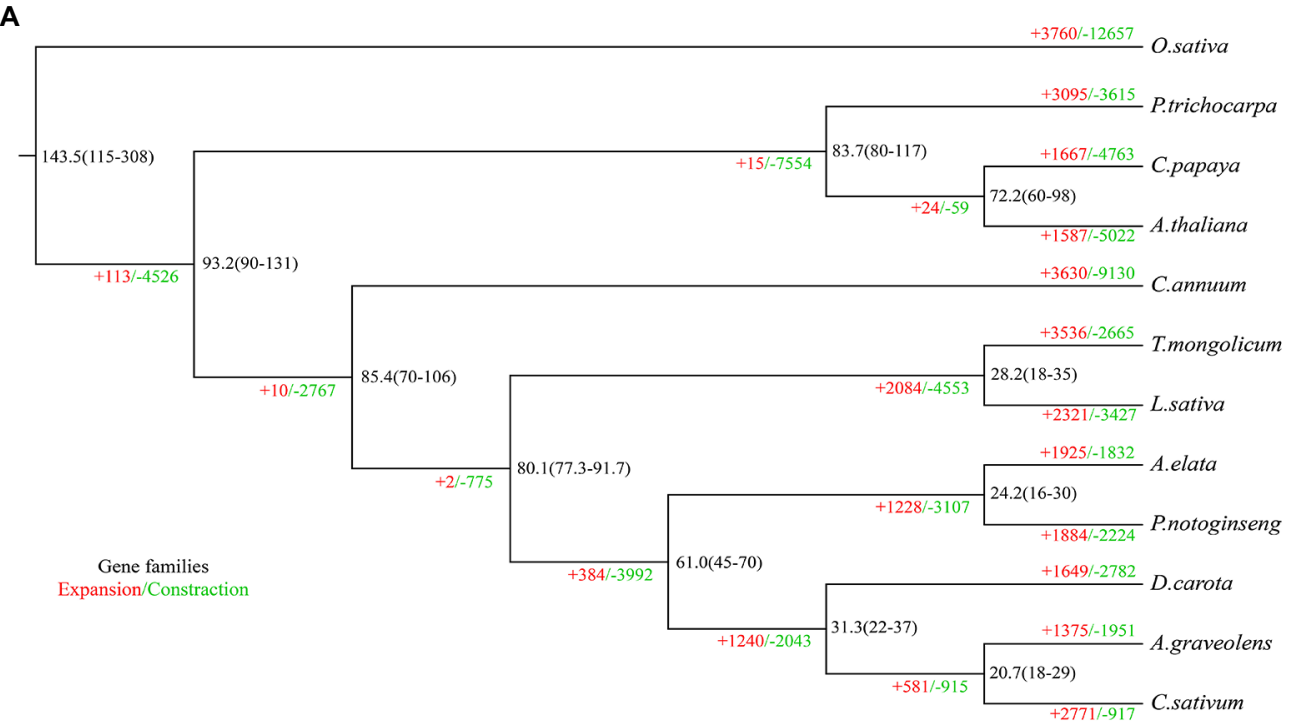

B

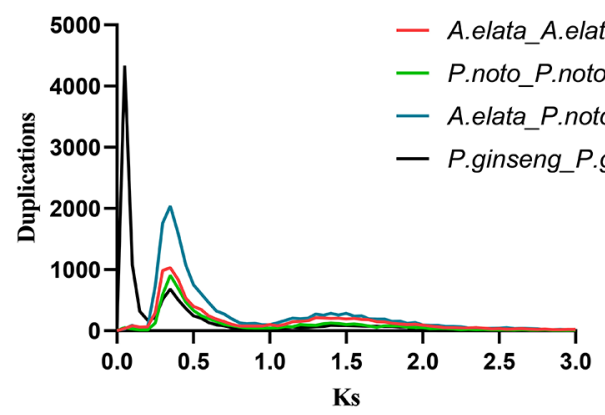

C

D

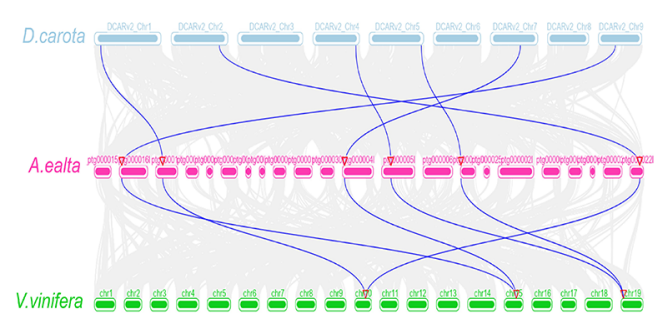

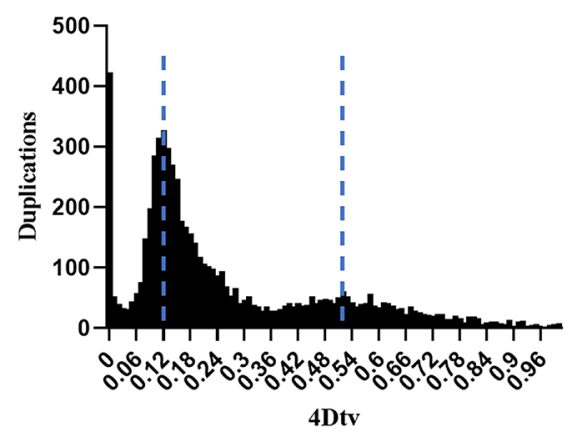

E

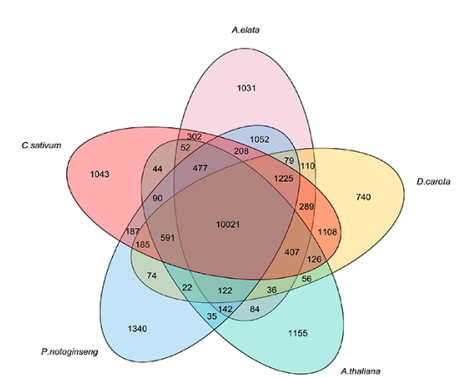

FIGURE 2 | (A) Inferred phylogenetic tree of Aralia elata and 11 plant species based on protein sequences of single-copy orthologous genes. Numbers at each node represent the estimated divergence time of each node in million years ago (mya). Gene family expansions are indicated in red, and gene family contractions are indicated in green. (B) Ks distribution of paralogous gene pairs in the A. elata, Panax notoginseng, and Panax ginseng genome. The probability density of Ks was estimated using the "density" function in the R language. (C) Distribution diagram of 4DTv values. The dark black-filled part indicates the 4DTv analysis inside A. elata, and the peaks marked by the dotted line indicate where the two whole genome duplication (WGD) events of $A$. elata occurred. (D) Collinear analysis among Daucus carota, $A$. elata, and Vitis vinifera genome. The blue lines in the genomes of $A$. elata and $V$. vinifera indicate that the $2: 1$ correspondence between the two collinear regions. (E) Venn diagram showing the cluster distribution of shared gene families among $A$. elata, C. sativum, P. notoginseng, D. carota, and A. thaliana.

The gene family analysis among these species revealed that the 33,499 genes in the A. elata genome were clustered into 15,637 gene families with an average size of 2.14. The members in the gene families varied greatly, and the largest family contained 277 genes. We then investigated the specific and shared gene families among the species of A. elata, C. sativum,
P. notoginseng, A. thaliana, and D. carota. The results indicated that 10,021 gene families were observed in all the investigated species, and 1,031 gene families appeared to be lineage specific to A. elata (Figure 2E).

Whole genome duplication occurs widely in flowering plants and plays important roles in genome evolution, the formation 
of new species, and gene neofunctionalization (Piegu et al., 2006; Van de Peer et al., 2009). The previous results indicated that two species in Araliaceae, P. notoginseng and Panax ginseng, have experienced one and two recent WGD events, respectively (Kim et al., 2018; Jiang et al., 2021). To further explore the evolutional trajectory of $A$. elata, we investigated the WGD events in its genome. The protein sequences from the A. elata genome were searched against themselves using BLASTP $(\mathrm{E}<1 \mathrm{e}-5)$ to identify homologous gene pairs (Camacho et al., 2009). We calculated the 4DTv (4-fold degenerate synonymous sites of the third codons) for the optimal gene pairs and plotted the distribution of the $4 \mathrm{DTv}$ values (Figure 2C). Two peaks were observed at approximately 0.12 and 0.50 , respectively. The right peak at approximately 0.50 revealed the core eudicot gamma triplication event. The left peak at approximately 0.12 indicated that $A$. elata underwent a recent WGD event. We then investigated the syntenic blocks between $V$. vinifera and $A$. elata using McscanX to further confirm the WGD event in $A$. elata, because $V$. vinifera does not undergo any recent WGDs. A 2:1 syntenic relationship between A. elata and $V$. vinifera (Figure 2D) was observed, which confirmed the recent WGD event occurred in the A. elata genome.

Ks (synonymous substitution rate) values can be used to estimate the timing of large-scale duplications (Blanc and Wolfe, 2004). We calculated the $K s$ values of the gene pairs and plotted the distributions to estimate the occurrence time of the WGD events of $A$. elata, $P$. notoginseng, and P.ginseng, respectively (Figure 2B; Chen et al., 2020). Two peaks were observed in the Ks distributions of the $P$. notoginseng and $A$. elata genomes, whereas the P.ginseng genome contained three $K s$ peaks. The Ks distribution result of $A$. elata was consistent with the $4 \mathrm{DTv}$ values. The main peak at approximately 0.38 indicated that a recent WGD event occurred in the A. elata genome. Similar Ks peaks around 0.38 were also found in the $P$. notoginseng and P.ginseng genomes, which indicated that the recent WGD event may be shared by A. elata, P. notoginseng, and P.ginseng. Then we calculated the occurring time of the WGD event of $A$. elata according to the method reported (Qin et al., 2014). The WGD event was estimated to occur at approximately 29.6 mya in the A. elata genome. Because the divergence time of $A$. elata and $P$. notoginseng was estimated to be 24.2 mya, this WGD event may occur before the differentiation of the two species. This is consistent with the published result (Jiang et al., 2021). All the results above indicated that unlike $P$. ginseng, who experienced an extra genus specific WGD, A. elata and $P$. notoginseng genome experienced only one recent WGD (Kim et al., 2018). In addition, this WGD may be shared by species in Araliaceae.

\section{Analysis of Key Enzyme Encoding Genes Involved in Triterpene Saponins Biosynthesis}

The biosynthesis pathways of terpenoids in plants have been comprehensively explained. The research on Aralia Linn. plants have attracted extensive interest from researchers. By integrating sequence similarity, conserved domain, and phylogenetic relationship results (Figure 3; Supplementary Figure 2; Supplementary Table 8), we identified 22 candidate genes encoding the enzymes that may catalyze the biosynthesis processes of terpenoids in the A. elata genome (Figure 4). We used transcriptome sequencing data of $A$. elata downloaded from public database to investigate the expressional profiles of these genes. The RNA-seq reads were aligned to the genome assembly and obtained their expression levels in roots, stems, and leaves. Figure 4 illustrates the normalized expressional levels of these enzyme-coding genes in each tissue. The results indicated that many genes appeared to be expressed in tissuespecific manners. For example, genes encoding CYP450s are abundant in stems and roots.

Previous studies have shown that CYP72A and CYP716A subfamily members are the main CYP450s involved in the biosynthesis of pentacyclic triterpene saponins. Therefore, we pay special attention to the four CYP716a and three CYP72a coding genes identified in the A. elata genome. At the same time, 12 genes related to the terpene skeleton and triterpene biosynthesis pathway were identified, including MVA pathway and 2,3-oxysqualene biosynthesis pathway. Among them, six enzymes (AACT, HMGS, HMGR, MK, PMK, and MVD) were associated with the MVA pathway.

In the MVA pathway, Acetyl-CoA is synthesized into Acetoacetyl-CoA, which is catalyzed by the AACT enzyme (encoded by Arel.002085). The expression level of Arel.002085 in leaves and roots are slightly higher than that in stems. Acetoacetyl-CoA is synthesized into 3-Hydroxy-3methylglutaryl-CoA, which is catalyzed by HMGS enzyme (encoded by Arel.020097). HMGR enzyme (encoded by Arel.004178) subsequently catalyzes the synthesis of Mevalonic acid, and then MK enzyme (encoded by Arel.022041) is used to catalyze the synthesis of Mevalonic acid-5P. The expressional levels of Arel.020097, Arel.004178, and Arel.022041 in roots were higher than those in stems and leaves, indicating that the biosynthetic reaction mainly occurred in the roots of $A$. elata. Then, under the catalysis of PMK enzyme (encoded by Arel.027136), Mevalonic acid-5-pyrophosphate was generated, and then Isopentenyl pyrophosphate is catalyzed by MVD enzyme (encoded by Arel.003662), and then catalyzed by IPPI enzyme (encoded by Arel.030181) to form Dimethylallyl pyrophosphate, further condensation of Isopentenyl pyrophosphate and Dimethylallyl pyrophosphate to form various terpenoids. Except for Arel.003662, whose expression levels in leaves and roots are slightly higher than those in stems, the expression levels of Arel.027136 and Arel.030181 in stems are slightly higher than those in leaves and roots. The results indicated that these biosynthetic reactions in this part may occur in the stems.

After that, Isopentenyl pyrophosphate and Dimethylallyl pyrophosphate is catalyzed by GPS enzyme (encoded by Arel.001014) to produce Geranyl diphosphate. FPS enzyme (encoded by Arel.030122) then catalyzes Geranyl diphosphate into Farnesyl pyrophosphate. SS enzyme (encoded by Arel.022914) catalyzes Farnesyl pyrophosphate into Squalene. Finally, 2,3-oxidosqualene is synthesized by the catalysis of SE enzyme (encoded by Arel.013186). In the synthetic pathway, Arel.001014 


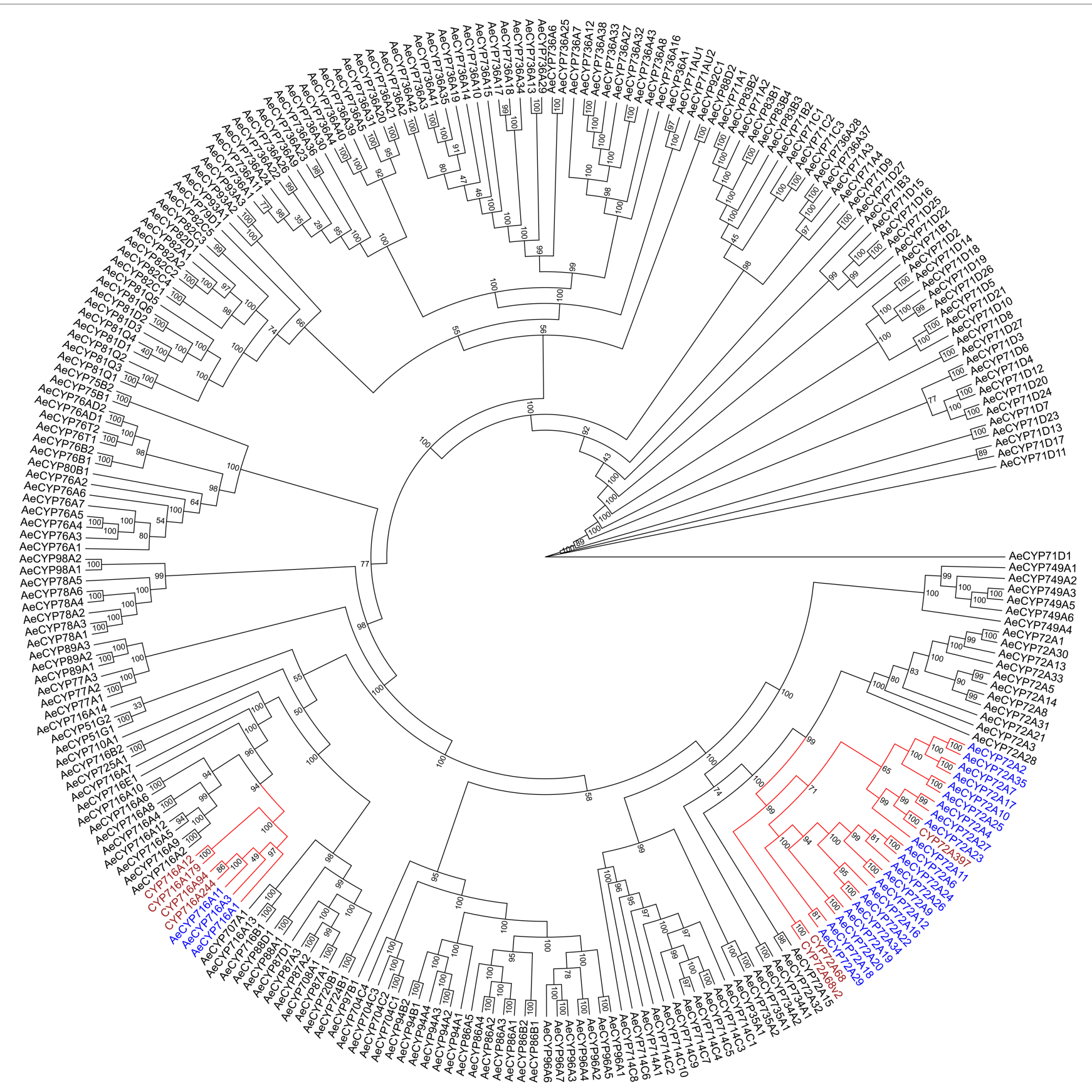

FIGURE 3 | Phylogenetic analysis of P450 genes. Genes that putatively encode Cytochromes P450 were identified by the integration of sequence similarities, conserved domains, and the phylogeny. The colored clades in the phylogenetic tree include genes encoding the subfamilies of CYP72A and CYP716A, which are the key enzymes of P450 participated in the biosynthetic pathway of triterpene saponins. Genes marked in purple are reported to catalyze the synthesis of triterpene saponins in other species.

and Arel.030122 genes are expressed at higher levels in leaves and stems, while Arel.002914 and Arel.013186 genes are expressed at higher levels in stems and roots. Based on these results, it is speculated that the key triterpene skeleton biosynthesis reaction mainly occurs in the stem.

Finally, 2,3-oxidosqualene is catalyzed by $\beta$-AS (encoded by Arel.032339, Arel.032335, and Arel.020446) to form $\beta$-amyrin, and then oleanolic acid is formed under the catalysis of CYP716A subfamily members (including CYP716A244, CYP716A12, CYP716A179, and CYP716A94), and then Hederagenin is formed under the catalysis of CYP72A subfamily members (including CYP72A68, CYP72A68v2, and CYP72A397). Among them, genes encoding CYP72A and CYP716A subfamily members have higher expression levels in roots and stems than in leaves. 

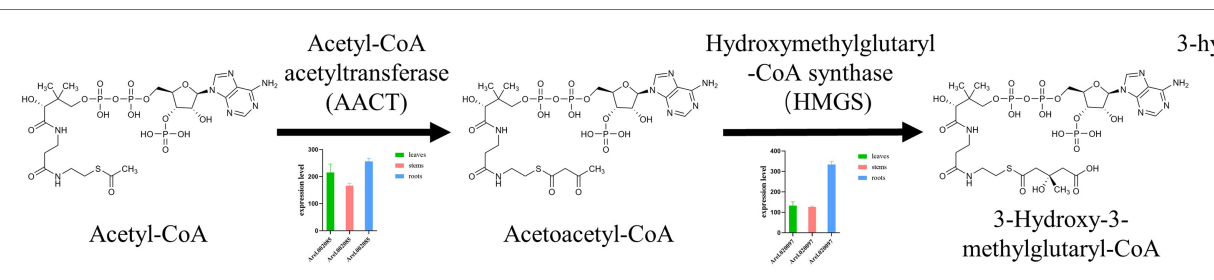

3-hydroxy-3-methylglutaryl-

CoA reductase

(HMGR)
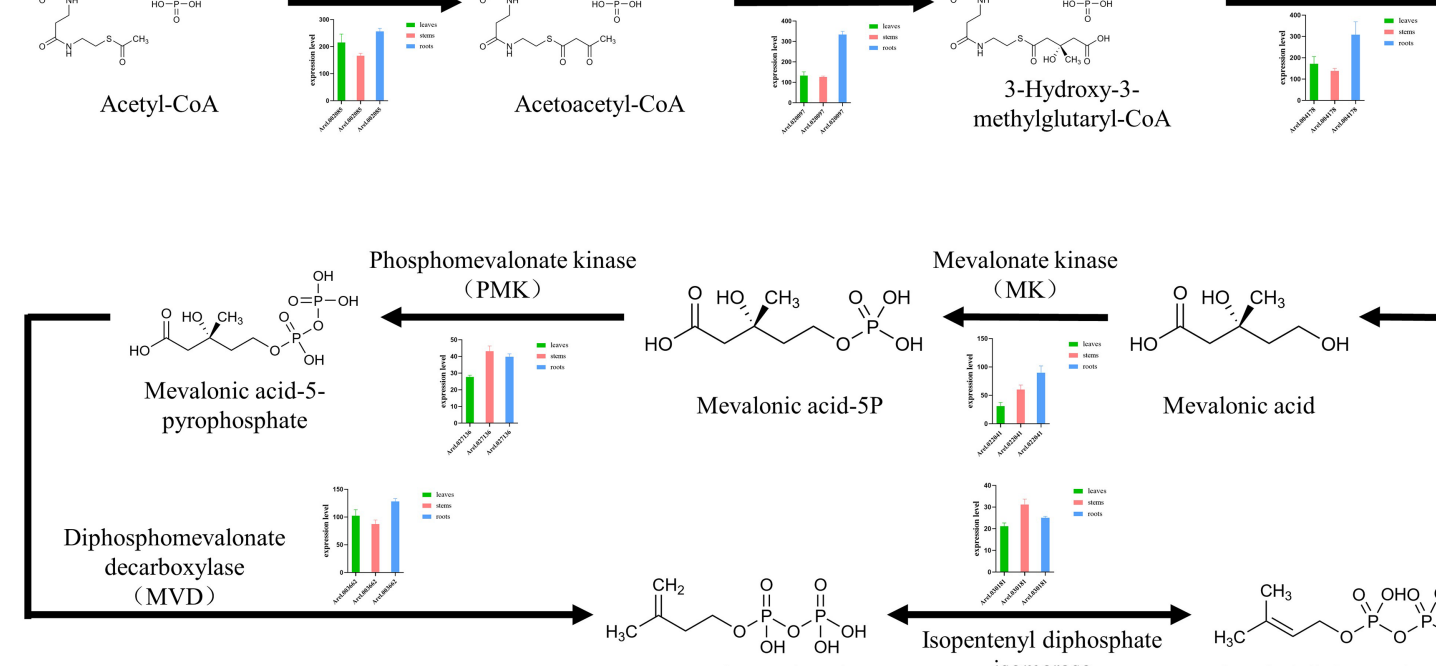

Mevalonate kinase

Isopentenyl pyrophosphate

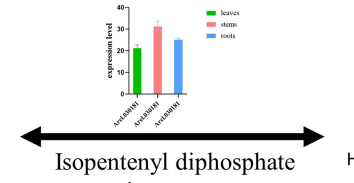

(MK)

$\leftarrow$ =둥

Int.".

$=\mathrm{HO}_{\mathrm{OH}}$

Mevalonic acid

Isopentenyl diphosphate

isomerase

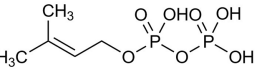

Dimethylallyl pyrophosphate

Geranyl diphosphate
synthase

(GPS)

Farnesyl diphosphate synthase
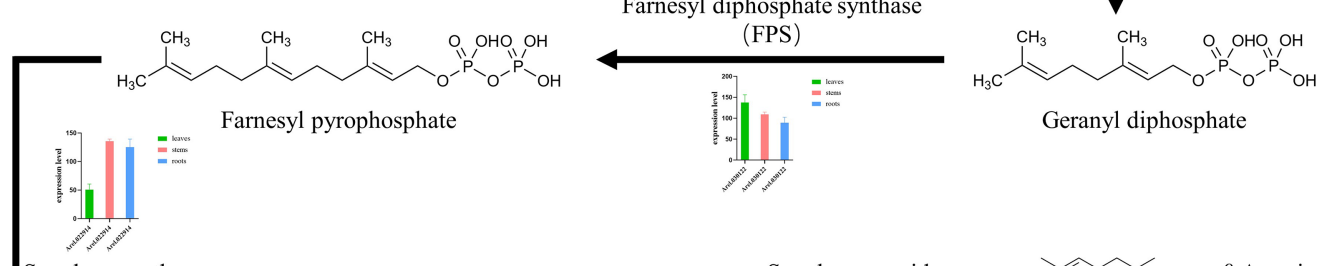

Geranyl diphosphate

Squalene synthase
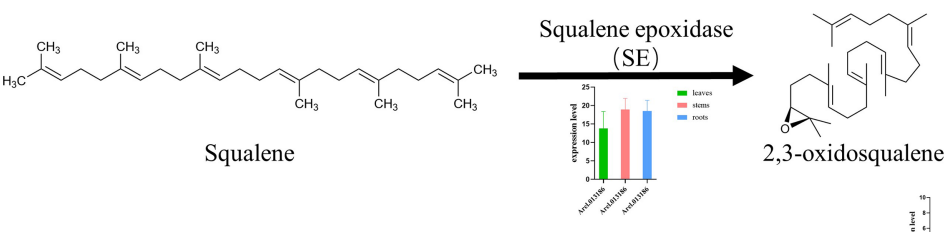

$\beta$-Amyrin synthase

Squalene

CYP716A244

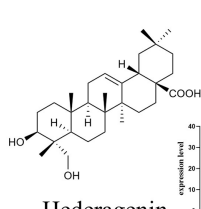

Hederagenin
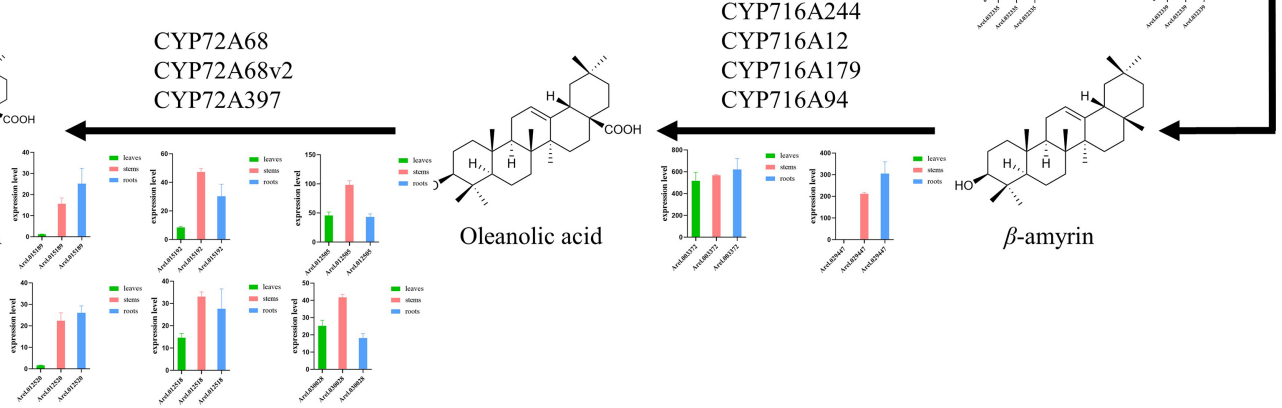

FIGURE 4 I Overview of the saponin biosynthetic pathway in Aralia elata and expression profiles of key enzyme encoding genes. The histogram shows the expression levels of different genes in different tissues. Green indicates the amount of expression in the leaves, red indicates the amount of expression in the stem, and blue indicates the amount of expression in the root. The most genes of enzymes involved in the saponin biosynthesis pathway were highly expressed in roots higher than in leaves and stems.

Based on the expression levels of these genes, we explored the secondary metabolism in A. elata plants at the spatial levels. We compared the expressional patterns of these genes in different tissues. We found that most of the genes involved in the saponin biosynthesis were specifically expressed in roots, and a few were highly expressed in leaves and stems (Figure 5). 


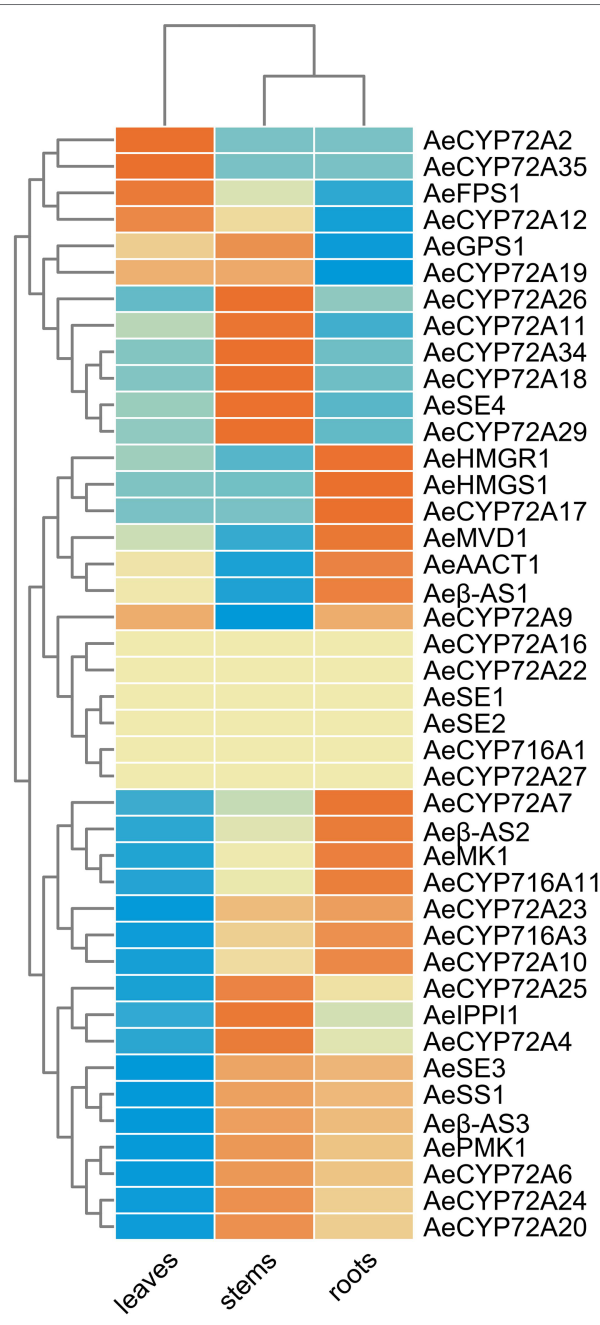

FIGURE 5 | Heatmap of gene transcript abundance in the saponin biosynthetic pathway of key enzyme genes at three different parts in Aralia elata. RPKM values are log2-based. Yellow and blue indicate high and low expression levels, respectively.

\section{Establishment of the Agrobacterium Mediated Transformation System for Aralia elata}

Biotechnology is an efficient way to increase the contents of secondary metabolites in plants. The annotation of $A$. elata genome will provide many candidate genes for the generation of genetically modified A. elata plants. However, it is still difficult for genetic transformation in A. elata. Based on the embryonic callus induction system of $A$. elata established in our laboratory, we set up the genetic transformation system of this plant. Roots of well-grown tissue culture seedlings were used as explants for A. tumefacien infestation, and the roots were precultured, co-cultured, and selection cultured (kanamycin resistance) to obtain resistant callus. DNA extracted from the resistant callus was examined by PCR. As shown in Figure 6, the target fragment was successfully detected in the positive transgenic plants and found to be better transformed at $10 \mathrm{~min}$

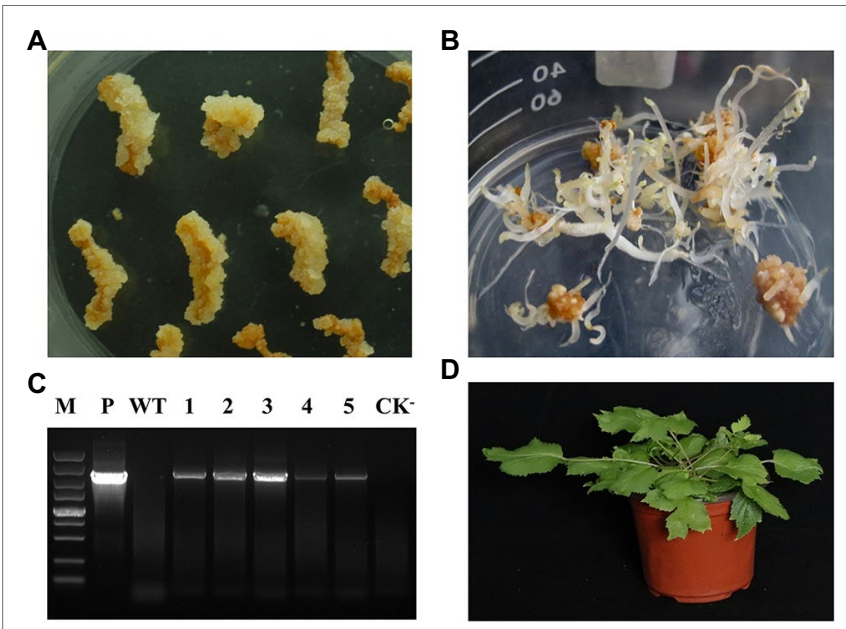

FIGURE 6 | Establishment systems of the propagation and gene information of Aralia elata. (A) The induction of the somatic embryogenic callus from the root in the $1 / 2 \mathrm{SH}$ medium with $3.0 \mathrm{mg} / \mathrm{L}$ of IBA and $0.2 \mathrm{mg} / \mathrm{L}$ of $\mathrm{KT}$ for

3-week culture. (B) The somatic embryogenesis from that callus in the $1 / 2 \mathrm{SH}$ medium with $1.0 \mathrm{mg} / \mathrm{L}$ of IBA and $0.2 \mathrm{mg} / \mathrm{L}$ of $\mathrm{KT}$ for 3 -week culture.

(C) Introduction of the exogenous genes in the plant. P, positive control; The WT was not transformed with Agrobacterium; 1-5, the transgenic plant with target gene; $\mathrm{CK}^{-}$, negative control. (D) The somatic embryo plant of gene transformation.

of infection time. We transferred the transgenic callus to differentiation medium to obtain somatic embryonic seedlings. Next, the somatic embryo seedlings were transferred to WPM medium containing $20 \mathrm{~g} / \mathrm{L}$ sucrose and cultured under $16 \mathrm{~h}$ light and $8 \mathrm{~h}$ dark conditions for 4 weeks, and then the plants were moved into soil and cultured in a greenhouse for 2 months, as shown in Figure 6, the transgenic plants grew well, and finally we obtained transgenic plants.

\section{DISCUSSION}

Aralia elata is one of the most widely used Chinese medicinal plants from the family Aralialeae and is well known in China and worldwide for its good efficacy. Triterpenoid saponins are widely existed in Aralialeae and are the most studied active ingredients of $A$. elata. Most of the aglycones are oleanolic acid, ivy, and their derivatives. Kochetkoy (1963) reported its chemical composition for the first time and obtained three saponins. The research on saponins of $A$. elata has become a hot topic, and many studies have reported its chemical components. Up to now, more than 100 saponins have been isolated and identified from A. elata. However, the complete biosynthetic pathway of saponins of $A$. elata has not been determined and further research is needed. Here, we briefly analyzed the terpenoid biosynthesis pathway of A. elata, to provide a reference for follow-up research. The contents of triterpenoid saponins in A. elata could be increased by genetically modification of the candidate genes involved in this pathway. The annotated genome and the genetic transformation system established in this study would be used for the further functional genome analysis in this species. 
In the family Aralialeae, the genomes of some species including Eleuthorcoccus senticusus (Yang et al., 2021), P. ginseng (Kim et al., 2018), and P. notoginseng (Jiang et al., 2021) have been reported. The high-quality genomic analysis of $A$. elata will provide a valuable extensive information for studying the evolutionary landscape of other species in Araliaceae. Gene mining of high-quality genomic and transcriptomic data can provide resources for further exploration of plant growth and secondary metabolism mechanisms (Tu et al., 2020). So, we produced the first high-quality genome reference for $A$. elata with the latest sequencing technologies and bioinformatics methods. The size of the assembled genome is very close to the predicted result of $K$-mer, reflecting no obvious expansion or collapse occurred during the assembly process. Benefit from the long lengths and high accuracy of HiFi reads, the continuity and completeness of the $A$. elata genome obtained in this study are at a high-quality level. The evolutional process of the genome was studied based on the genome. Our results combined with the published genomes revealed the WGD trajectory in Araliaceae. A recent WGD event occurred before the divergence of species in Araliaceae.

In conclusion, the high-quality $A$. elata genome sequence described in this article, combined with comparative genome analysis, identification and tissue species expression analysis of putative genes involved in saponins biosynthesis, and the establishment of an efficient genetic transformation system of $A$. elata will contribute to $A$. elata breeding and cultivation.

\section{DATA AVAILABILITY STATEMENT}

The datasets presented in this study can be found in online repositories. The names of the repository/repositories and

\section{REFERENCES}

Ahn, D. (1998). Illustrated Book of Korean Medicinal Herbs. Seoul (Korea): Kyo-hak Publishing Co., 107.

Blanc, G., and Wolfe, K. H. (2004). Widespread paleopolyploidy in model plant species inferred from age distributions of duplicate genes. Plant Cell 16, 1667-1678. doi: 10.1105/tpc.021345

Camacho, C., Coulouris, G., Avagyan, V., Ma, N., Papadopoulos, J., Bealer, K., et al. (2009). BLAST+: architecture and applications. BMC Bioinformatics 10:421. doi: 10.1186/1471-2105-10-421

Carelli, M., Biazzi, E., Panara, F., Tava, A., Scaramelli, L., Porceddu, A., et al. (2011). Medicago truncatula CYP716A12 is a multifunctional oxidase involved in the biosynthesis of hemolytic saponins. Plant Cell 23, 3070-3081. doi: 10.1105/tpc.111.087312

Chen, C., Chen, H., Zhang, Y., Thomas, H. R., Frank, M. H., He, Y., et al. (2020). TBtools: an integrative toolkit developed for interactive analyses of big biological data. Mol. Plant 13, 1194-1202. doi: 10.1016/j.molp.2020.06.009

Cheng, H. (2011). Study on Genes Related to Triterpenoid Saponin Biosynthesis Pathway in Aralia elata. ChangChun, China: Jilin University.

Cheng, H., Concepcion, G., Feng, X., Zhang, H., and Li, H. (2008). Haplotyperesolved de novo assembly with phased assembly graphs. arXiv. [Preprint].

Cheng, Y., Liu, H., Tong, X., Liu, Z., Zhang, X., Chen, Y., et al. (2021). Effects of shading on triterpene saponin accumulation and related gene expression of Aralia elata (Miq.) seem. Plant Physiol. Biochem. 160, 166-174. doi: 10.1016/j.plaphy.2021.01.009 accession number(s) can be found in the article/ Supplementary Material.

\section{AUTHOR CONTRIBUTIONS}

$\mathrm{XY}$ conceived the project. XY and SuC designed the experiments. WL, SoC, and WG performed most of the experiments and analyzed the data. The other authors assisted in the experiments and discussed the results. XY, SuC, WL, and WG wrote the manuscript. All authors contributed to the article and approved the submitted version.

\section{FUNDING}

This work was financially supported by the National Natural Science Foundation of China (No. 30972390) and the Fundamental Research Funds for the Central Universities (2572018CL02).

\section{ACKNOWLEDGMENTS}

The authors extremely appreciate the suggestions and comments from the editors and reviewers for improving the quality of this manuscript.

\section{SUPPLEMENTARY MATERIAL}

The Supplementary Material for this article can be found online at: https://www.frontiersin.org/articles/10.3389/fpls.2022.822942/ full\#supplementary-material

Cheng, Y., Liu, H., Tong, X., Liu, Z., Zhang, X., Li, D., et al. (2020). Identification and analysis of CYP450 and UGT supergene family members from the transcriptome of Aralia elata (Miq.) seem reveal candidate genes for triterpenoid saponin biosynthesis. BMC Plant Biol. 20:214. doi: 10.1186/ s12870-020-02411-6

Collu, G., Unver, N., Peltenburg-Looman, A. M., Van Der Heijden, R., Verpoorte, R., and Memelink, J. (2001). Geraniol 10-hydroxylase1, a cytochrome P450 enzyme involved in terpenoid indole alkaloid biosynthesis. FEBS Lett. 508, 215-220. doi: 10.1016/S0014-5793(01)03045-9

Dai, J.-L., Tan, X., Zhan, Y.-G., Zhang, Y.-Q., Xiao, S., Gao, Y., et al. (2011). Rapid and repetitive plant regeneration of Aralia elata seem. via somatic embryogenesis. Plant Cell Tissue Organ Cult. 104, 125-130. doi: 10.1007/ s11240-010-9801-x

Darriba, D., Posada, D., Kozlov, A. M., Stamatakis, A., Morel, B., and Flouri, T. (2020). ModelTest-NG: a new and scalable tool for the selection of DNA and protein evolutionary models. Mol. Biol. Evol. 37, 291-294. doi: 10.1093/ molbev/msz189

De Bie, T., Cristianini, N., Demuth, J. P., and Hahn, M. W. (2006). CAFE: a computational tool for the study of gene family evolution. Bioinformatics 22, 1269-1271. doi: 10.1093/bioinformatics/btl097

Di Tommaso, P., Chatzou, M., Floden, E. W., Barja, P. P., Palumbo, E., and Notredame, C. (2017). Nextflow enables reproducible computational workflows. Nat. Biotechnol. 35, 316-319. doi: 10.1038/nbt.3820

Duan, H., Wang, D., Wang, S., and Wu, S. (2019). Research progress of Aralia continentalis kitag of bioactive components. Farm Product. Process. 
Emms, D. M., and Kelly, S. (2019). OrthoFinder: phylogenetic orthology inference for comparative genomics. Genome Biol. 20, 1-14. doi: 10.1186/ s13059-019-1832-y

Ewels, P. A., Peltzer, A., Fillinger, S., Patel, H., Alneberg, J., Wilm, A., et al. (2020). The nf-core framework for community-curated bioinformatics pipelines. Nat. Biotechnol. 38, 276-278. doi: 10.1038/s41587-020-0439-x

Fukushima, E. O., Seki, H., Ohyama, K., Ono, E., Umemoto, N., Mizutani, M., et al. (2011). CYP716A subfamily members are multifunctional oxidases in triterpenoid biosynthesis. Plant Cell Physiol. 52, 2050-2061. doi: 10.1093/ pcp/pcr146

Guo, X., Fang, D., Sahu, S. K., Yang, S., Guang, X., Folk, R., et al. (2021). Chloranthus genome provides insights into the early diversification of angiosperms. Nat. Commun. 12, 1-14. doi: 10.1038/s41467-021-26922-4

Haas, B. J., Papanicolaou, A., Yassour, M., Grabherr, M., Blood, P. D., Bowden, J., et al. (2013). De novo transcript sequence reconstruction from RNA-seq using the trinity platform for reference generation and analysis. Nat. Protoc. 8, 1494-1512. doi: 10.1038/nprot.2013.084

Haas, B. J., Salzberg, S. L., Zhu, W., Pertea, M., Allen, J. E., Orvis, J., et al. (2008). Automated eukaryotic gene structure annotation using EVidenceModeler and the program to assemble spliced alignments. Genome Biol. 9, 1-22. doi: $10.1186 / \mathrm{gb}-2008-9-1-\mathrm{r} 7$

Han, J.-Y., Kim, H.-J., Kwon, Y.-S., and Choi, Y.-E. (2011). The Cyt P450 enzyme CYP716A47 catalyzes the formation of protopanaxadiol from dammarenediol-II during ginsenoside biosynthesis in Panax ginseng. Plant Cell Physiol. 52, 2062-2073. doi: 10.1093/pcp/pcr150

Heitz, T., Widemann, E., Lugan, R., Miesch, L., Ullmann, P., Désaubry, L., et al. (2012). Cytochromes P450 CYP94C1 and CYP94B3 catalyze two successive oxidation steps of plant hormone jasmonoyl-isoleucine for catabolic turnover. J. Biol. Chem. 287, 6296-6306. doi: 10.1074/jbc.M111.316364

Höfer, R., Dong, L., André, F., Ginglinger, J.-F., Lugan, R., Gavira, C., et al. (2013). Geraniol hydroxylase and hydroxygeraniol oxidase activities of the CYP76 family of cytochrome P450 enzymes and potential for engineering the early steps of the (seco) iridoid pathway. Metab. Eng. 20, 221-232. doi: 10.1016/j.ymben.2013.08.001

Iorizzo, M., Ellison, S., Senalik, D., Zeng, P., Satapoomin, P., Huang, J., et al. (2016). A high-quality carrot genome assembly provides new insights into carotenoid accumulation and asterid genome evolution. Nat. Genet. 48, 657-666. doi: 10.1038/ng.3565

Irmler, S., Schröder, G., St-Pierre, B., Crouch, N. P., Hotze, M., Schmidt, J., et al. (2000). Indole alkaloid biosynthesis in Catharanthus roseus: new enzyme activities and identification of cytochrome P450 CYP72A1 as secologanin synthase. Plant J. 24, 797-804. doi: 10.1046/j.1365-313x.2000.00922.x

Jiang, Z., Tu, L., Yang, W., Zhang, Y., Hu, T., Ma, B., et al. (2021). The chromosomelevel reference genome assembly for Panax notoginseng and insights into ginsenoside biosynthesis. Plant Commun. 2:100113. doi: 10.1016/j.xplc.2020.100113

Kim, N. H., Jayakodi, M., Lee, S. C., Choi, B. S., Jang, W., Lee, J., et al. (2018). Genome and evolution of the shade-requiring medicinal herb Panax ginseng. Plant Biotechnol. J. 16, 1904-1917. doi: 10.1111/pbi.12926

Kochetkoy, H. K. (1963). Chemical constituent of Aralia elata. DoklAkadNauk 50:1289.

Kumar, S., Stecher, G., Suleski, M., and Hedges, S. B. (2017). TimeTree: a resource for timelines, timetrees, and divergence times. Mol. Biol. Evol. 34, 1812-1819. doi: $10.1093 / \mathrm{molbev} / \mathrm{msx} 116$

Li, H. (2013). Aligning sequence reads, clone sequences and assembly contigs with BWA-MEM. arXiv [Preprint].

Marchler-Bauer, A., Lu, S., Anderson, J. B., Chitsaz, F., Derbyshire, M. K., Deweese-Scott, C., et al. (2010). CDD: a conserved domain database for the functional annotation of proteins. Nucleic Acids Res. 39, D225-D229. doi: $10.1093 /$ nar/gkq1189

Nafisi, M., Goregaoker, S., Botanga, C. J., Glawischnig, E., Olsen, C. E., Halkier, B. A., et al. (2007). Arabidopsis cytochrome P450 monooxygenase $71 \mathrm{~A} 13$ catalyzes the conversion of indole-3-acetaldoxime in camalexin synthesis. Plant Cell 19, 2039-2052. doi: 10.1105/tpc.107.051383

Nguyen, L.-T., Schmidt, H. A., Von Haeseler, A., and Minh, B. Q. (2015). IQ-TREE: a fast and effective stochastic algorithm for estimating maximum- likelihood phylogenies. Mol. Biol. Evol. 32, 268-274. doi: 10.1093/molbev/ msu300

Piegu, B., Guyot, R., Picault, N., Roulin, A., Saniyal, A., Kim, H., et al. (2006). Doubling genome size without polyploidization: dynamics of retrotranspositiondriven genomic expansions in Oryza australiensis, a wild relative of rice. Genome Res. 16, 1262-1269. doi: 10.1101/gr.5290206

Qin, C., Yu, C., Shen, Y., Fang, X., Chen, L., Min, J., et al. (2014). Wholegenome sequencing of cultivated and wild peppers provides insights into capsicum domestication and specialization. Proc. Natl. Acad. Sci. U. S. A. 111, 5135-5140. doi: 10.1073/pnas.1400975111

Reunov, A., Reunova, G., and Zhuravlev, Y. N. (2007). Morphological study of pollen grains in mature anthers of Aralia elata, A. continentalis, and A. cordata (Araliaceae). Dokl. Biol. Sci. 417, 465-468. doi: 10.1134/ s0012496607060166

Robinson, M. D., and Oshlack, A. (2010). A scaling normalization method for differential expression analysis of RNA-seq data. Genome Biol. 11:R25. doi: 10.1186/gb-2010-11-3-r25

Sawai, S., and Saito, K. (2011). Triterpenoid biosynthesis and engineering in plants. Front. Plant Sci. 2:25. doi: 10.3389/fpls.2011.00025

Simão, F. A., Waterhouse, R. M., Ioannidis, P., Kriventseva, E. V., and Zdobnov, E. M. (2015). BUSCO: assessing genome assembly and annotation completeness with single-copy orthologs. Bioinformatics 31, 3210-3212. doi: 10.1093/ bioinformatics/btv351

Tu, L., Su, P., Zhang, Z., Gao, L., Wang, J., Hu, T., et al. (2020). Genome of Tripterygium wilfordii and identification of cytochrome P450 involved in triptolide biosynthesis. Nat. Commun. 11:971. doi: 10.1038/s41467-020-14776-1

Van De Peer, Y., Maere, S., and Meyer, A. (2009). The evolutionary significance of ancient genome duplications. Nat. Rev. Genet. 10, 725-732. doi: 10.1038/ $\operatorname{nrg} 2600$

Vurture, G. W., Sedlazeck, F. J., Nattestad, M., Underwood, C. J., Fang, H., Gurtowski, J., et al. (2017). GenomeScope: fast reference-free genome profiling from short reads. Bioinformatics 33, 2202-2204. doi: 10.1093/bioinformatics/ btx 153

Wu, Y. (2011). Clong and Fundational Analysis of Triterpenoid Saponin Related Genes in Aralia elata. ChuangChun,China: Jilin University.

Yang, Z. (2007). PAML 4: phylogenetic analysis by maximum likelihood. Mol. Biol. Evol. 24, 1586-1591. doi: 10.1093/molbev/msm088

Yang, Z., Chen, S., Wang, S., Hu, Y., Zhang, G., Dong, Y., et al. (2021). Chromosomal-scale genome assembly of Eleutherococcus senticosus provides insights into chromosome evolution in Araliaceae. Mol. Ecol. Resour. 21, 2204-2220. doi: 10.1111/1755-0998.13403

Zhang, Y., Wang, W., He, H., Song, X.-Y., Yao, G.-D., and Song, S.-J. (2018). Triterpene saponins with neuroprotective effects from a wild vegetable Aralia elata. J. Funct. Foods 45, 313-320. doi: 10.1016/j.jff.2018.04.026

Zhao, C. Y. (2012). Clong and Genetic Transfermaiton of Key Genes Related to Triterpenoid Saponin Biosynthetic Pathway in Aralia elata. ChuangChun, China: Jilin University.

Conflict of Interest: The authors declare that the research was conducted in the absence of any commercial or financial relationships that could be construed as a potential conflict of interest.

Publisher's Note: All claims expressed in this article are solely those of the authors and do not necessarily represent those of their affiliated organizations, or those of the publisher, the editors and the reviewers. Any product that may be evaluated in this article, or claim that may be made by its manufacturer, is not guaranteed or endorsed by the publisher.

Copyright (c) $2022 \mathrm{Liu}, \mathrm{Guo}$, Chen, Xu, Zhao, Chen and You. This is an openaccess article distributed under the terms of the Creative Commons Attribution License (CC BY). The use, distribution or reproduction in other forums is permitted, provided the original author(s) and the copyright owner(s) are credited and that the original publication in this journal is cited, in accordance with accepted academic practice. No use, distribution or reproduction is permitted which does not comply with these terms. 